\title{
Teaching - Learning Strategies in Interactive Education - A Case Study
}

\author{
V Bharathi Harishankar ${ }^{1}$, Archana Ghode ${ }^{3}$, Alankar Bandyopadhayay ${ }^{2}$, \\ Maryma Scotlin ${ }^{3}$, and Hema A Murthy ${ }^{3}$, Timothy A Gonsalves ${ }^{3}$, Ashok \\ Jhunjhunwala ${ }^{3}$ \\ 1 Department of English, Institute of Distance Education, University of \\ Madras \\ 2 University of Maryland \\ 3 TeNeT Group, IIT Madras
}

\begin{abstract}
This paper discusses active teaching and learning strategies that have been used in the TeNeT Online tutorials [1]. The focus of these tutorials is to enable rural students to pass the Standard X examination. The highlight of the tutorial is the use of the Question and Answer format. This problem-solving approach is evident in its three-pronged format: Learn, Practice and Test. Every module is based on questions and answers. In the learn mode, students learn the steps to answer a question and thus the concepts behind it. In the practice and evaluation modes, students can test their knowledge and understanding. This approach enhances the element of interactivity in these tutorials. Combined together, the elements of interactivity and problem-solving have made the tutorials a healthy supplement to conventional class rooms.
\end{abstract}

Keywords: web-based, interactivity, learn, practice, test, problem-solving

\section{Introduction}

The dynamics of teaching and learning usually involves three entities: teacher, domain of knowledge and student. The Internet has today become the fourth entity. The Internet has made available information at the click of a mouse. It is being increasingly used as an important resource in distance learning [2-6]. What is key in these initiatives is the inclusion of interactivity. In fact, feedback on MIT's [2] initiative has been that most youngsters would like more interactivity.

The TeNeT Online tutorials correspond to a similar experiment at the high school level. Since the target audience are high school students, the emphasis in material has been to keep the interactivity as high as possible. This is achieved by using a problemsolving approach to learning. The paper is organized as follows: Section 2 briefly 
outlines the aims and goals of the tutorials. Section 3 analyses the overall design features of the tutorials. Section 4 elaborates the three main features of our tutorials namely Learn, Practice and Test sections. Section 5 examines the problem-solving approach that has been enhanced in this method. Section 6 gives details on field trials of the tutorials. Section 7 concludes on an optimistic note in terms of scalability of effort.

\section{Background to TeNeT Online Project}

The TeNeT online project was initiated with the express purpose of enabling rural students in Tamil Nadu to pass the Standard X examinations. A random survey revealed that the percentage of failures was very high in English followed by Maths, Science and Social Studies in that order. One possible reason is that for students of vernacular medium, English is a subject taught within the confines of the classroom and not a language used prevalently in day to day communication. This is compounded by the fact that most rural students are first generation learners and do not have a support system at home (parents/tuition teachers) to supplement the material taught at school. The inevitable fallout of this is that rural students are rarely encouraged by their parents to repeat the exams again. Very often, they go back to the family trades and professions. More importantly, this failure keeps them out of more lucrative jobs because even shop floor jobs require a minimum pass in Standard X.

TeNeT Online's first set of material was a solved question bank with ten question papers each for English papers 1 and 2 of the Tamil Nadu board. The small initial feedback showed a marked improvement in marks obtained in class room tests. It also showed that there was demand for similar materials in other subjects, namely, Maths, Science and Social Studies. The primary goal of the TeNeT Online Tutorial is to supplement class room teaching. Another goal of the tutorial is to reach uniform teaching material to a large number of rural students. This is the primary reason for making the material web-based. Further the tutorials are able to provide individual attention to a student as opposed to a teacher who has to cater to 120 children in a high school. The obvious caution we took is to avoid making the online course "an electronic page turning" or "an audio-visual aid to rote learning." A major shift in focus that we have effected through interactivity is to transform the approach from conceptual learning to one of problem solving.

\section{Design Strategies in TeNeT Online}

Against this background, our goal was to provide, an easy to use interface. The following criteria were kept in mind when we design the tutorials:

1. gaining the attention of the students

2. stimulating recall

3. providing learning guidance 
4. providing instant feedback

5. assessing performance

6. enhancing retention and transfer

In our tutorials, we combined a variety of commonly known software. Our program combines HTML and Java script at the front end and MySQL at the backend with PHP providing the link between the two. Different features of our tutorials combine to achieve the various steps in the instruction process. These include voice-overs, visual pop ups, gifs, mouse-overs, highlighting of key phrases and division of the web page in to main and work areas. This has resulted in the on line tutorials becoming guided educational explorations. These features have been combined in different fashions in the three main divisions of our tutorials, namely learn, practice and Test.

\section{Main Components of TeNeT Online}

A typical module of our tutorial provides three options to the learner: learn, practice, test. Of these, learn and practice are presented simultaneously as 2 links on the same page so that students can easily switch between learning and self-testing mode.

\subsection{Learn mode}

In this mode, the student comes across a question and its answer, If it is a multiple choice question, the answer is highlighted in a different color. A click on the "Play" (Figure 1) button enables the student to listen to the teacher's voice guiding them through the different steps to solve a particular question.

In subjects like Maths, different steps carry marks in the final exam. Key words and phrases in each answer are highlighted. In common practice, we don't recall entire answers from a book in xerox fashion but remember phrases/ points (Figure 2) which become memory cues. (Eg. Science and Social Studies especially, formulae, concepts, definitions, years, etc.)

In a subject like English, the learn mode offers a bilingual experience. The page that is viewed is in English whereas the voice overs are in the local language.

Apart from enabling understanding, it allows the students to relate words 


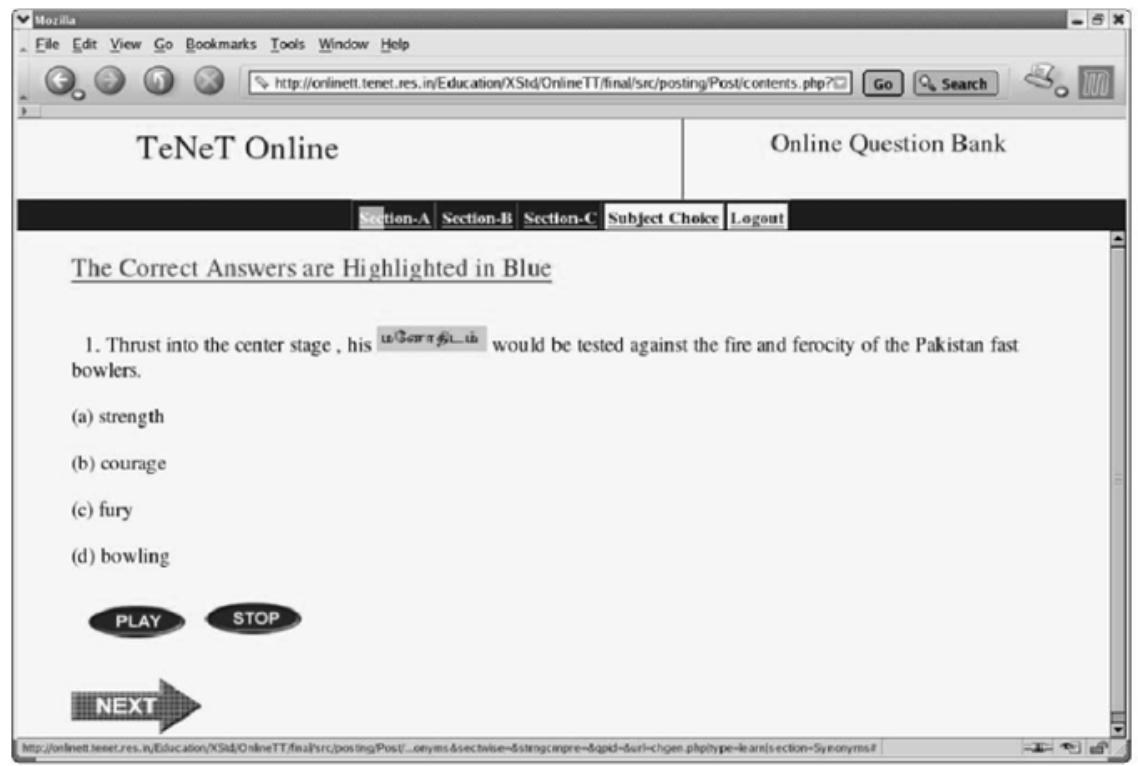

Figure 1: Multiple choice questions

to their meanings. Moreover, difficult words are highlighted and on mouse over, the meaning appears in the local language (Figure 1,2)

Some picture gifs are also introduced to provide variety to the text. The underlying rationale for all these features is to maintain a high instructor profile as also to preempt possible queries and doubts.

\subsection{Practice Mode}

In this mode, students get an opportunity to assess themselves on what they have learnt. This apparently simple format is, in fact, the key to the interactivity of the module. For instance, once the student makes the choice, the voice over says 'correct' or 'wrong' (Figure 3) 


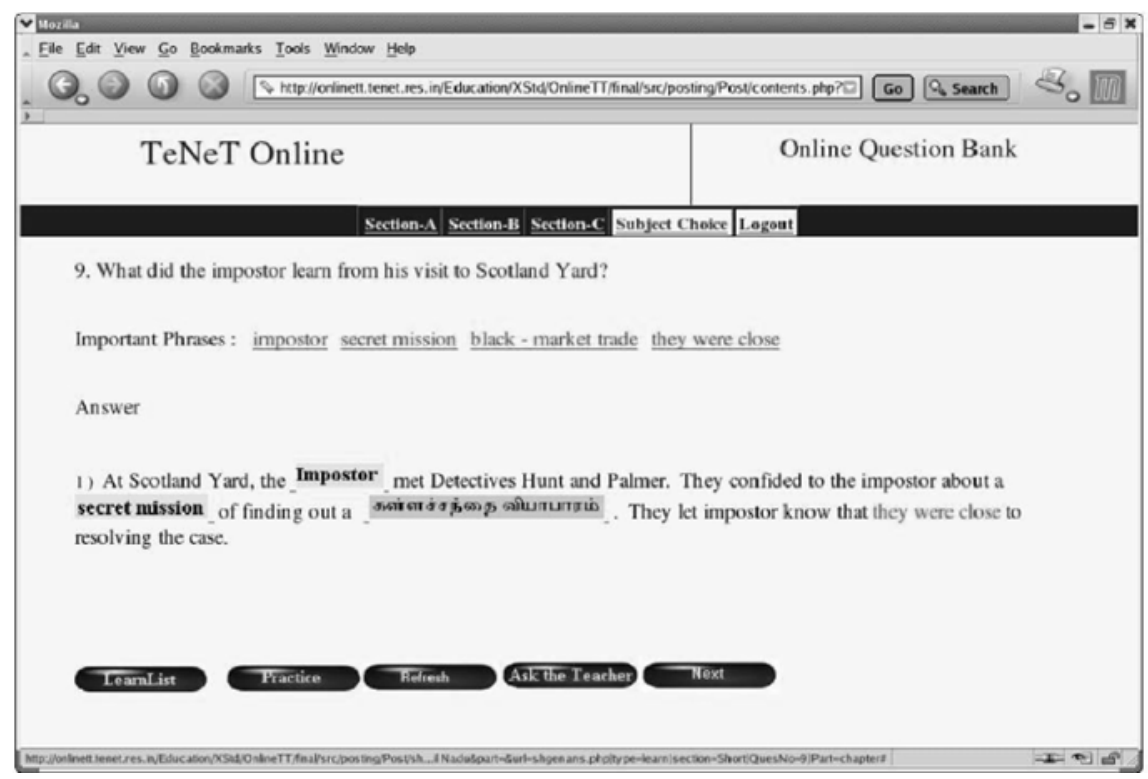

Figure 2: Keywords and phrases

Simultaneously, a picture gif projects a laughing face for the correct answer and a crying face for the wrong answer Figure 3). Apart from allowing students to know their mistakes, some modules, like our Social Science module, provide a clue as to where the correct answer may be found in the text book.

Since the online tutorial is almost always taken by a group of students (say 4 or 5), the practice module offers "collaborative or group learning" [7] by which an answer may be discussed, corrected or guessed without any penalty of marks or punishment and justified by the students themselves. This aspect is rarely encouraged in typical class room conditions and is therefore a highlight of our modules. The objective is to develop a testing environment with instant feedback to students.

Typically, every answer has key phrases. In the practice mode, the key phrases are given with several options in listboxes and the student makes a choice to click the correct answer. This mode teaches the students the step-by-step ordering of an answer. It is extremely useful in a subject like Maths, where every correct step is apportioned some marks.

By dividing the page into two halves, we follow an instant display of the correct and wrong options. This is done through the use of font colours. For instance, the 'red' display indicates a wrong choice and 'blue' indicates the correct choice. Moreover, it alerts the students to understand that in practice, if they go wrong in step 1, they cannot reach step 2 and so on. Indirectly, this weans them away from memorising the answers and to a minimal extent work on a cause and effect model. 
We have also found that students become "picture perfect," by practice as in the use of maps in social science and diagrams in science. These two types of questions are perennially present in every board exam question paper. Instead of hazarding a guess, just by visually going over the page again and again, students can master these exercises. For instance, a sure question in Social Studies is the map question, in which students are expected to accurately point out the different places, regions and rivers on the given map (Figure 4).

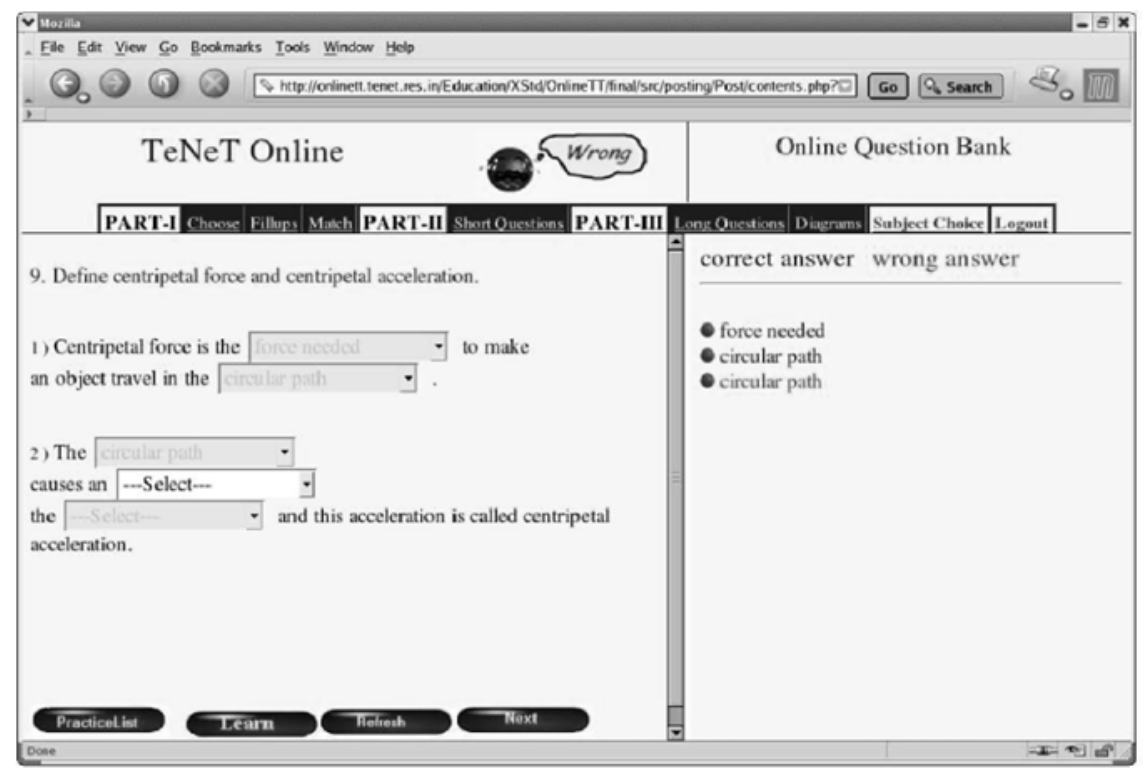

Figure 3: Practice Mode

In this mode, we provide a map with a lot of hot keys. After choosing the name of the place, students have to click on the correct location. This provides an interesting way of learning to mark the maps. Students also realize that it is not enough to know the location roughly and that a difference of even a millimeter will render their marking incorrect. In the present model, everything from specific places, to regions and rivers are isolated single points. In our future course of work, we hope to create a program by which students can actually draw the course of the river, for instance.

\subsection{Test Mode}

In the test mode, students are given the choice of attempting an entire question paper, thus simulating an exam hall situation. A further step in our tutorial is the concept of automatic generation of papers. This ensures that students receive a new question paper, every time they access it. It also simulates the pattern their final examination 
paper will take. For example, a typical computer generated question paper takes in to account the weightage given to each chapter, choices available, answer keys used by examiners to correct the answers and the like. Our final evaluation apportions marks for each section, by which a students can judge her/his own weaknesses and strengths. They will also be able to compare their earlier performances, overall and in individual sections (Figure 5).

\section{Figure 4: Map marking}

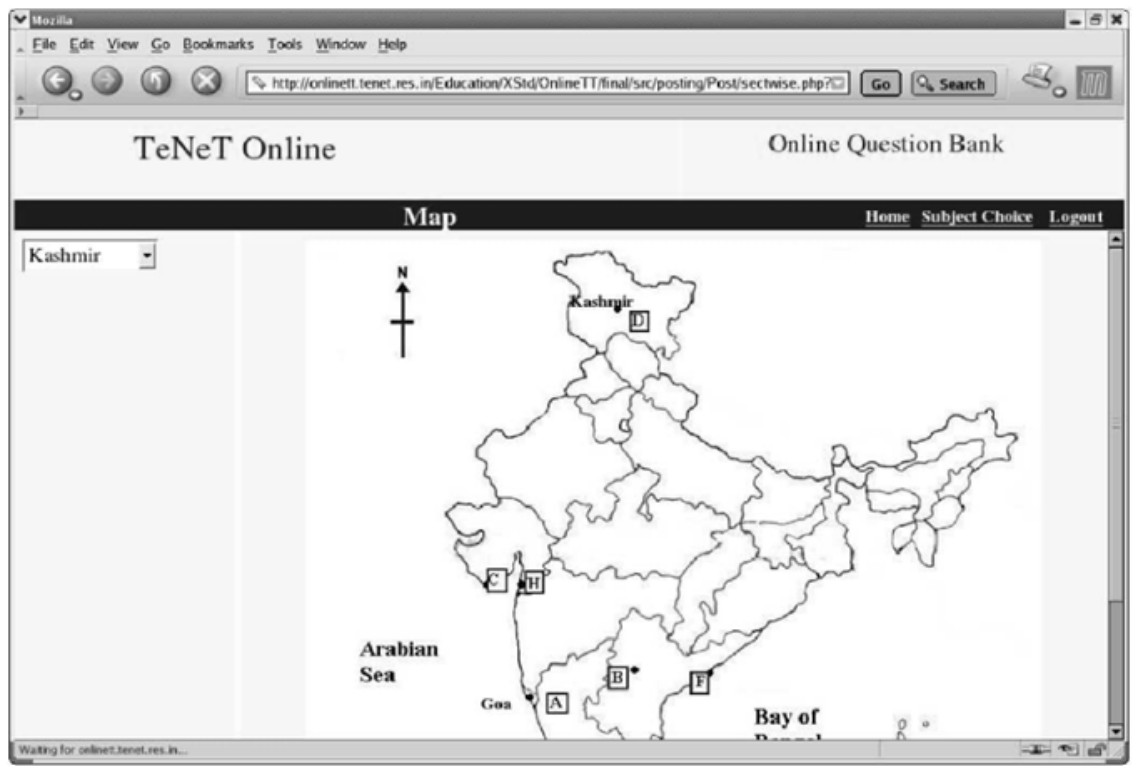

\section{Problem Solving Approach in TeNeT Online}

The focus of this paper and our online tutorial is to consider design aspects that can help improve the instructional effectiveness of teaching and learning. By designing an interactive instructional environment, the tutorials have taken into account two main factors, namely, the ways in which an information is processed and how learners perceive the process, store and recall information. Further, the interactivity is not achieved through tools like email, list servers, chat rooms or news groups, but through a dynamism worked in to the format of the web page itself. The effort here is to transform the student from being a"passive recipient of knowledge towards being a student involved in the learning process as an active, self- directed participant" [8].

The online tutorials reinforce a problem-solving approach to teaching and 
learning. For instance, there are certain common types of questions in each paper. These include: Fill in the blanks, Choose the best answer, Match the following, Short questions and Long questions. At a surface level, each of these questions seems to demand a different answer. However, they are all taken from the same lessons or chapters. Therefore, we don't necessarily have to learn the same chapter differently for different questions. Once we understand the key ideas, we use different combinations to tackle different questions.

For all practical purposes, we have made key words and phrases as important elements in an answer. While this makes the students word perfect in objective type of questions like Fill ups, Choose and Match, it also offers them hints to develop and answer the short and long questions. For example, a student cannot be expected to know all the words in all the answers. Quite dangerously, this makes them resort to memorizing and short circuit understanding in the bargain. Moreover, when a question is slightly modified, students do not understand the question, let alone know the answer. [In the English Online Tutorial, students had to be told repeatedly that a question like What are dikes? is different from Why are dikes built? This does not mean that there are no overlaps. Both questions have to tackle the definition of a dike! There after, one describes the dike whereas the other explains its use.] In our method, we emphasise on the student learning and understanding the key words and phrases. Due to the fear of exams, even if they forget all the words, their memory of the key phrases will still fetch them marks. More importantly, we have followed the key phrase method in all the subjects. Thus, remembering an idea is not subject specific (i.e., it is not only for formulae or definitions), but is the mark of good learning. Different types of questions have been standardized in our method through the multiple choice format. This, according to us, is a win-win format because students fully understand the key phrases in any answer and online evaluation is made simple in this yes-no format of evaluation. 


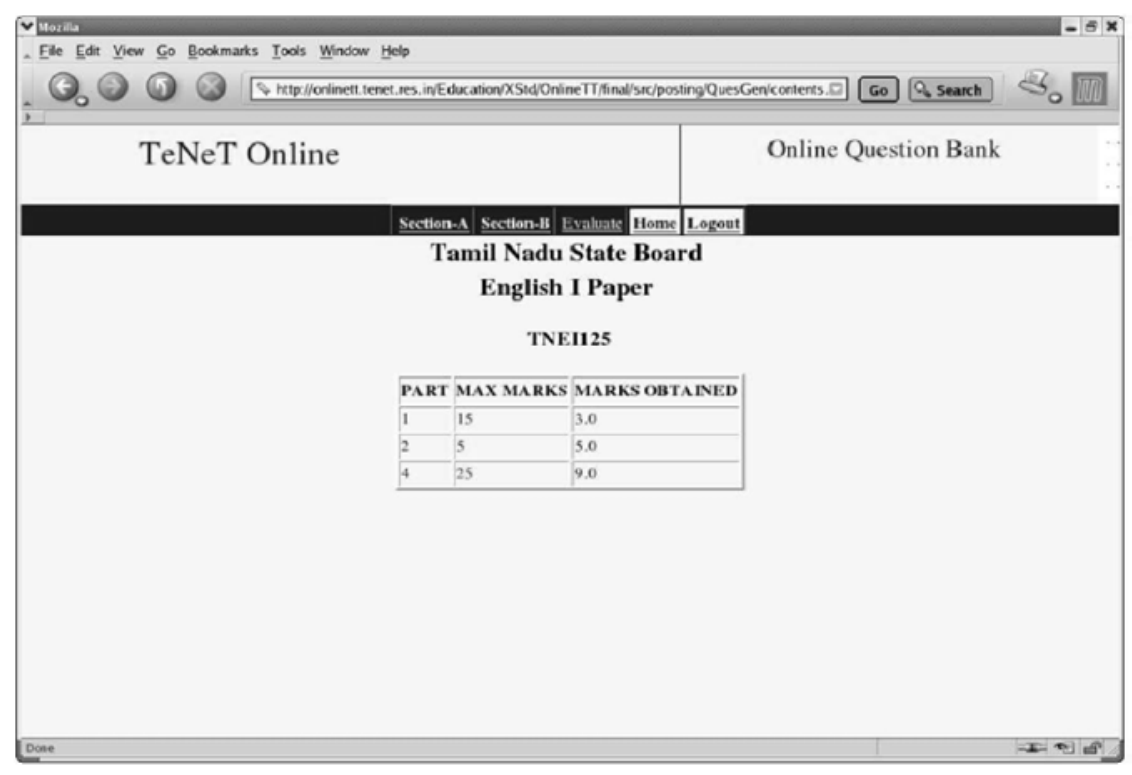

Figure 5: Test Results

\section{Field Trials and Monitoring}

The TeNeT online tutorials have been tested in the field for over two years now. The material was deployed in four centres in the first year, and in 18 centres in the second year. An NGO provided a scholarship for students to use this service. During the first year of deployment about 168 students went through the tutorials while during the second year about 800 students went through the tutorials. In both years, the performance in the final board exams has been complimentary. About $82 \%$ of the rural students passed the exam although the average for the entire state was about $75-77 \%$. Internet centre wise, it was observed that centres that had good moderators to present the material performed well. This clearly indicates that the material can be only used to supplement instruction and not replace it. In many villages, the school teachers encouraged the students to take the tutorials as it helped them perform better in the school internal exams.

To test the effectiveness of the tutorial reports are automatically generated which detail the usage at every Internet-centre. This includes information namely, number of hours used, details of subjects that were used, details of questions attempted (Figure 6). We also have details of students that are registered for the program (photo-ids of the students registered are available). Ideally one would like to use technology to automatically take the attendance at every session. Further, we also use videoconferencing to interact with students and monitor progress. In addition to this we also conduct mock examinations in 
all the four subjects over four days. The automatically generated question papers are printed and distributed to the various Internet centres. The students answer the papers as they would in an exam. These papers are the centrally corrected and feedback is provided to the students on their performance. We have found that our mock exams (conducted two months before the state board exam) have been very effective in helping the students prepare better for the final exam. Further, the mock exams are taken by all students (includes students who have not registed for the tutorials). In the last two years, we have observed that the students who registered for the tutorial performed significantly better than the students who didnot register for the tutorial.

\section{Conclusion}

TeNeT Online tutorials is an attempt to supplement teaching in high schools. The most important aspect of the tutorials seems to be the practice and test sessions that enables the students to get ready for the exams. Another important aspect of the tutorial is that it enables repetitive learning. Further this year, we have been able scale up to 200 Internet-Centres. Recently this material has been made available for standard XII too. There seems to be a lot of enthusiasm in the field with a large number of students, teachers and administrators getting interested. An important aspect of the tutorials is that it provides a uniform teacher for all students irrespective of village, town, city or school that they are from. As the Internet is used as the medium for delivery of instruction, it was very easy to scale up from a mere 4 Internet-centres in the first year to 18 centres in the second year and to 200 centres this year. This year we have started reaching out to other states. We have started work on Standard X material for Karnataka and Gujarat.

\section{Acknowledgement}

This work was funded by the Intel Project: Driving Applications over Wireless in Rural India (ELE0304103INTIASHO/R430).

\section{References}

[1] http://onlinett.tenet.res.in

[2] http://www.ocw.mit.edu

[3] http://www.cmu.edu/oli

[4] http://ocw.usu.edu

[5[http://ocw.jhsph.edu

[6] http://ocw.tufts.edu

[7] Le Jeune, Noel F, "Paper on Collaborative Learning in WBI", 
http://www.ouray.cudenver.edu/nflejeun/doctoralweb/Courses/IT 5640.html

[8]Mathew, Norman and Maryanne Dobery-Poirier, "Using the WWW to Enhance Classroom Instruction" First Monday. Vol.5, number 3, March 2000 http://irstmonday.org/issues/issue 53/mathew/index.html

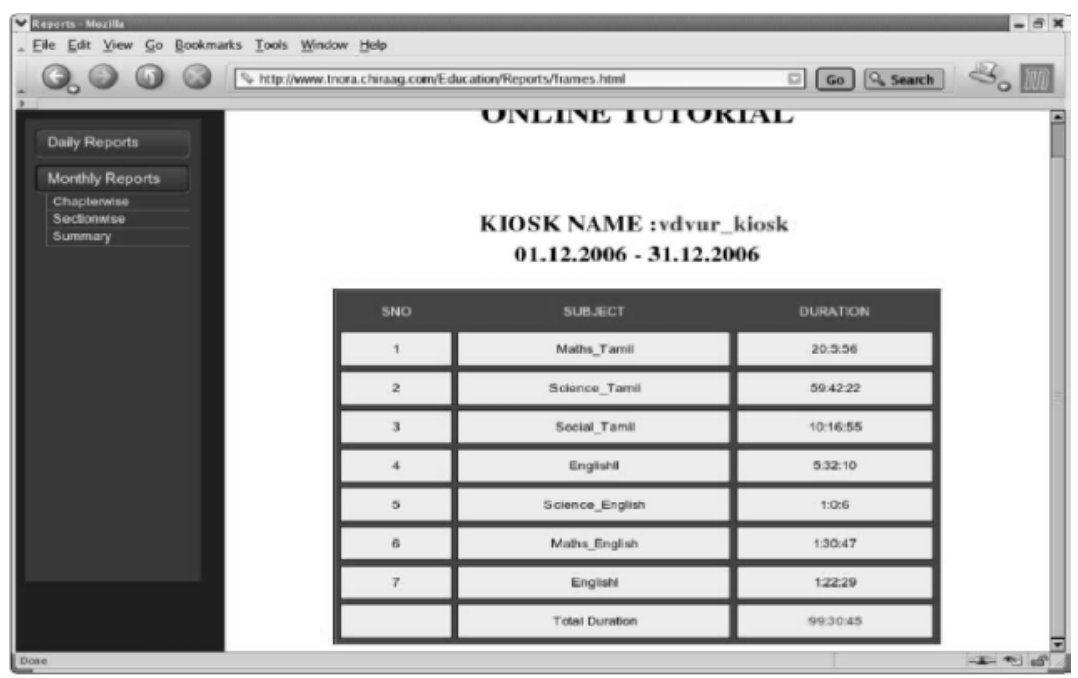

Figure 6: Usage Reports of an Internet Centre 